\title{
Vasoepididymostomy: an insight into current practice patterns
}

\author{
Ujval S. Pathak ${ }^{1}$, Adithya Balasubramanian ${ }^{1}$, Jonathan A. Beilan ${ }^{2,3}$, Mohit Butaney ${ }^{4}$, Alexander J. Tatem ${ }^{2,3}$, \\ Nannan Thirumavalavan ${ }^{2,3,5}$, Larry I. Lipshultz ${ }^{2,3}$ \\ ${ }^{1}$ Baylor College of Medicine, Houston, TX, USA; ${ }^{2}$ Scott Department of Urology, ${ }^{3}$ Center for Reproductive Medicine, Baylor College of Medicine, \\ Houston, TX, USA; ${ }^{4}$ Department of Urology, Mayo Clinic, Rochester, MN, USA; ${ }^{5}$ Urology Institute, University Hospitals/Case Western Reserve \\ School of Medicine, Cleveland, OH, USA \\ Contributions: (I) Conception and design: US Pathak, A Balasubramanian, JA Beilan, LI Lipshultz; (II) Administrative support: US Pathak, A \\ Balasubramanian, JA Beilan, AJ Tatem, N Thirumavalavan, LI Lipshultz; (III) Provision of study material or patients: None; (IV) Collection and \\ assembly of data: US Pathak, A Balasubramanian, JA Beilan, M Butaney, N Thirumavalavan, LI Lipshultz; (V) Data analysis and interpretation: US \\ Pathak, A Balasubramanian, JA Beilan, M Butaney, N Thirumavalavan, LI Lipshultz; (VI) Manuscript writing: All authors; (VII) Final approval of \\ manuscript: All authors. \\ Correspondence to: Larry I. Lipshultz, MD. Professor, Department of Urology, Baylor College of Medicine, 6624 Fannin Street, \#1700, Houston, TX \\ 77030, USA. Email: larryl@bcm.edu.
}

Background: Vasectomy reversal (VR) is a specialized procedure currently offered by an increasing number of medical practitioners. One method of VR, vasoepididymostomy (VE), is considered the most challenging microsurgical technique within the field of reproductive urology. We surveyed reproductive urologists to assess current practice patterns regarding both intra-operative and post-operative considerations surrounding $\mathrm{VE}$, with the hypothesis being that more experienced surgeons may have different practice patterns than less experienced surgeons.

Methods: An anonymous questionnaire was sent to members of the Society for Male Reproduction and Urology (SMRU). The survey included questions regarding case volume, preferred intra-operative techniques, and post-operative management strategies. Responses were collected using Survey Monkey (San Mateo, CA) and statistically analyzed with chi square tests.

Results: Three hundred and twenty SMRU members were contacted to participate in the survey; 74/320 (23.1\%) participants completed the survey in its entirety. Respondents performed varying amounts of VR annually with most surgeons (24\%) reporting between 11-20 VR per year and 15 surgeons (20.3\%) performed over 60 per year. Comparing practitioners who performed $\leq 30$ VR's annually $(n=46)$ to providers who performed $>30(\mathrm{n}=28)$ revealed a significantly lower rate of $\mathrm{VE}$ in low-volume practitioners $(\leq 20 \%$ vs. $>20 \%, \mathrm{P}<0.0001)$. The most commonly used technique to create the epididymotomy involved placing two 10-0 sutures into the tubule, followed by a sharp incision between the needles ( $74.3 \%$ of respondents). An intussusception anastomosis was the most commonly reported technique; $46.0 \%$ of participants utilize longitudinal stitch placement, while $35.1 \%$ place sutures horizontally. The most commonly reported time interval to evaluate the first post-reversal semen analysis (SA) was 6-8 weeks (39.2\%). Participants were also asked to rank the progression of adjunctive therapies employed in the setting of a subpar postreversal SA. Nonsteroidal anti-inflammatory drugs (NSAIDs) were the most popular first-line management option (52.7\%). Corticosteroids were the most frequently employed second-line option (37.8\%). Referral to an in vitro fertilization (IVF) center $(9.5 \%)$ and repeat surgery $(2.7 \%)$ were also options pursued by survey respondents. Most providers repeated the SA every 8-12 weeks (41.2\%) while following sub-par SA parameters.

Conclusions: VE is a technically demanding procedure that requires both microsurgical expertise and appropriate post-operative care. Our analysis demonstrates that a higher VR operative volume is associated with a higher rate of conversion to $\mathrm{VE}$. This indicates either more experienced surgeons are more likely to perform a VE when indicated or more experience surgeons are getting referred and/or performing more complex VRs. 
Keywords: Infertility; vasoepididymostomy (VE); vasectomy

Submitted Apr 26, 2019. Accepted for publication Sep 10, 2019.

doi: 10.21037/tau.2019.11.04

View this article at: http://dx.doi.org/10.21037/tau.2019.11.04

\section{Introduction}

Vasectomy reversal (VR) continues to grow in popularity since the development and advancement of microsurgical techniques in the 1970 s (1). Currently nearly $3-6 \%$ of patients who have undergone a vasectomy seek VR (2). Men pursue VR for several reasons including major life events such as divorce, death of a child or to relieve post-VR associated pain (1). VR was considered the only option to regain fertility following vasectomy until the emergence of assisted reproductive technologies (ART) (3). The procedure maintains several advantages over ART, including cost effectiveness, minimal hormonal manipulation of the female partner, and the ability to harvest sperm from ejaculate rather than surgical extraction via procedures such as microsurgical epididymal sperm aspiration (MESA).

Vasovasostomy (VV) and vasoepididymostomy (VE) are the two established techniques to achieve VR (4). Surgeons performing VV first remove the portion of the vas deferens that was sealed during the vasectomy procedure and develop patent ends on both the testicular and abdominal sides of the vas. Once the intervening portion is removed, vasal tubular fluid is expressed, sampled, and examined for opacity and viscosity as well as sperm microscopic content. If sperm heads are present in the vasal fluid under microscopic analysis, fluid conditions are deemed satisfactory and a VV is undertaken by surgically re-anastomosing the proximal and distal portions $(3,5,6)$. VE is indicated in cases where conventional VV may not result in successful VR. Several factors predict the need for VE, including epididymal obstruction, and concerning intraoperative findings such as the absence of sperm in the vasal fluid altogether (7). A prolonged time interval between vasectomy and VR is also a risk factor for VE (8). The exception is when copious clear fluid is found, even when devoid of sperm, vasovasostomy is indicated, and is usually successful.

$\mathrm{VE}$ is widely regarded as the most challenging microsurgical technique within the field of urology (9). Due to VE's technical complexity, it is critical that patients understand and characterize the surgical volume and expertise of their potential surgeon. Furthermore, it is crucial to identify when providers elect to undertake VE as opposed to VV. The aim of our study was to assess current practice patterns regarding both intra-operative and postoperative considerations surrounding VE to characterize the differences, if any, between experienced and less experienced surgeons.

\section{Methods}

An 8-question electronic survey was generated through Survey Monkey (San Mateo, California, USA) and sent to members of the Society for Male Reproduction and Urology (SMRU). SMRU is comprised of various healthcare stakeholders including urologists and andrologists. Three hundred and twenty SMRU members were invited to complete the survey. Participants provided consent prior to submitting their anonymous responses. The survey was designed to assess details about case volume, surgical technique, and post-operative practices in the setting of VR. The survey questions are available as supplementary information (Supplementary file 1). The survey was released 3 times and open for a 2-month period. Only surveys with complete responses for all questions were included for analyses. Descriptive analyses were performed, and chisquared analysis was used to determine the association between categorical variables. Statistical analyses were performed using Microsoft Excel (Redmond, Washington, USA).

\section{Results}

A total of 320 SMRU members were contacted to participate in the survey. Seventy-four (23.1\%) SMRU members completed the survey in its entirety over a 2-week period. The operative VR volume of survey respondents is summarized in Figure 1. Respondents included in this study performed various numbers of VR annually with most surgeons $(18 / 74,24.3 \%)$ performing between 11-20 VR per year. A majority of respondents (40/74, 54.1\%), indicated that they performed VE on one or both sides in $0-20 \%$ of their cases. Nearly half of respondents $(34 / 74,45.9 \%)$ 
Overview of number of vasectomy reversals performed by survey respondents

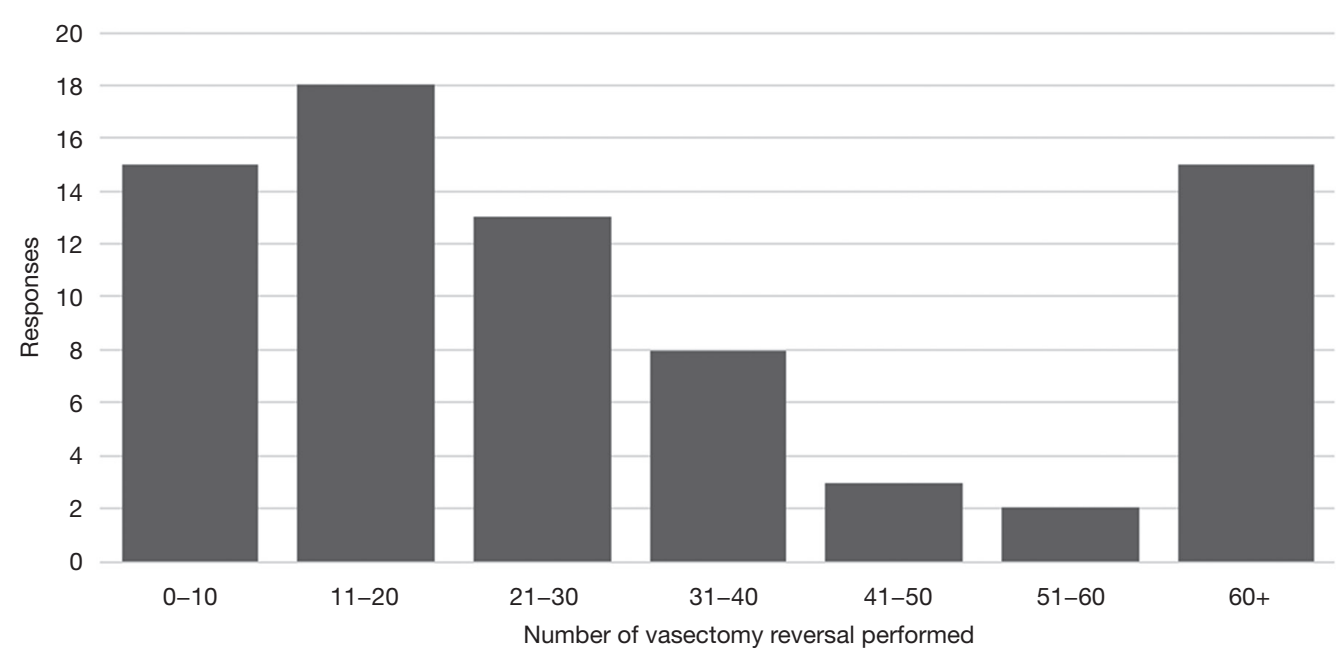

\begin{tabular}{|c|c|}
\hline Number of vasectomy reversals performed & Responses, $\mathrm{n}(\%)$ \\
\hline $0-10$ & $15(20 \%)$ \\
\hline $11-20$ & $18(24 \%)$ \\
\hline $21-30$ & $13(18 \%)$ \\
\hline $31-40$ & $8(11 \%)$ \\
\hline $41-50$ & $3(4 \%)$ \\
\hline $51-60$ & $2(3 \%)$ \\
\hline $60+$ & $15(20 \%)$ \\
\hline Total responses & 74 \\
\hline
\end{tabular}

Figure 1 Overview of number of vasectomy reversals performed by survey respondents.

undertook VE in 20-60\% of VR cases. Chi squared analyses revealed a statistically significant increase in the rate of performing VE in surgeons who performed more than 30 VR's annually $(\mathrm{n}=28)$ compared to providers who perform less than $30(\mathrm{n}=46)$ annually $(\mathrm{P}<0.0001)$.

Participants were queried upon preferred surgical technique to create an epididymotomy (Figure $2 A$ ). Most respondents $(55 / 74,74.3 \%)$ reported that they created an epididymotomy by placing two 10-0 sutures into the tubule, followed by a sharp incision between the needles. Participants were also queried upon preferred anastomotic surgical technique (Figure $2 \mathrm{~B}$ ). An intussusception anastomosis was the most commonly reported technique with $46.0 \%$ of respondents indicating that they utilize a longitudinal stitch placement, while $35.1 \%$ place sutures horizontally. Providers were also asked to identify the time period at which they order their first post-reversal semen analysis (SA) (Figure 3A). The most commonly reported time interval to evaluate the first post-reversal SA was $6-8$ weeks (29/74, 39.2\%). No significant association was present between case volume and epididymotomy and anastomotic surgical technique.

Participants were asked to rank the progression of adjunctive medical therapies they employ to improve subpar post-reversal SA. In the setting of a subpar postreversal SA, NSAIDs were the most popular first-line management option (52.7\%), followed by corticosteroids (23.0\%). Referral to an in vitro fertilization (IVF) center $(9.5 \%)$ or repeat surgery $(2.7 \%)$ were less popular options (Table 1). In the setting of subpar post-reversal SA parameters, respondents repeat the SA every 8-12 weeks (41.2\%) (Figure 3B). Statistically significant associations between case volume and post-operative management strategies were not present. 


\section{A}

Two 10-0 sutures into the tubule, with an incision to create the epididymotomy the

No sutures; a blade/microknife used to enter the tubule

Microscissors to open an ellipse in the tubule

Using a $10-0$ or 11-0 suture to pull through and create a hole in the tubule
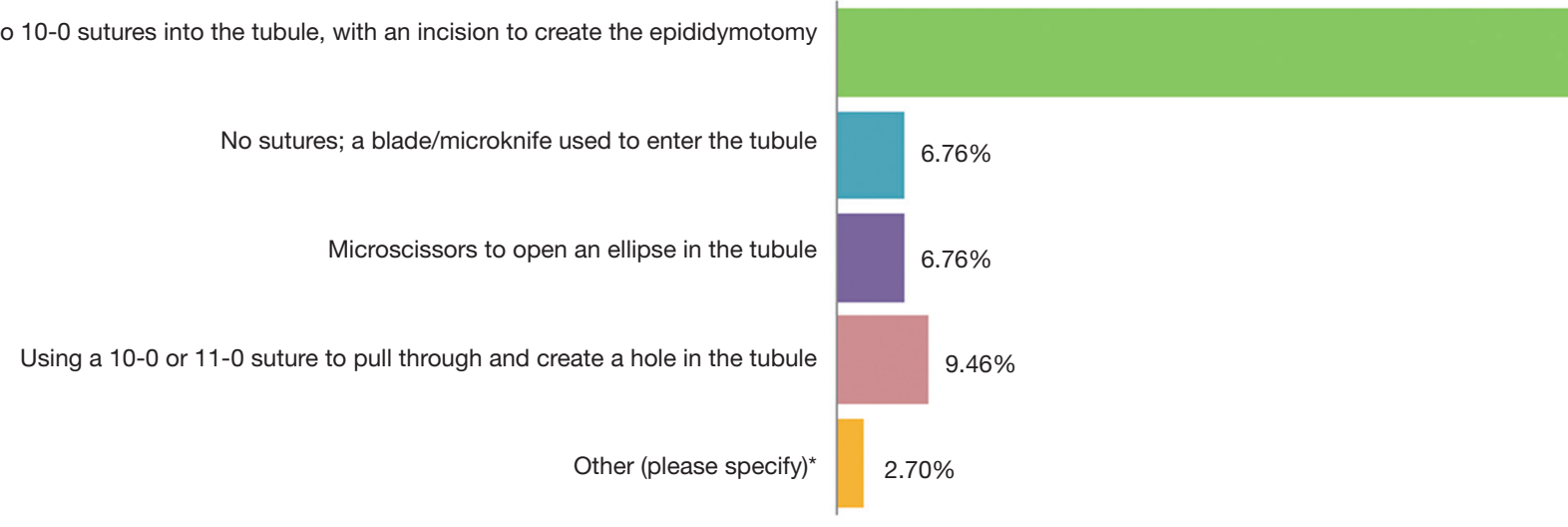

B

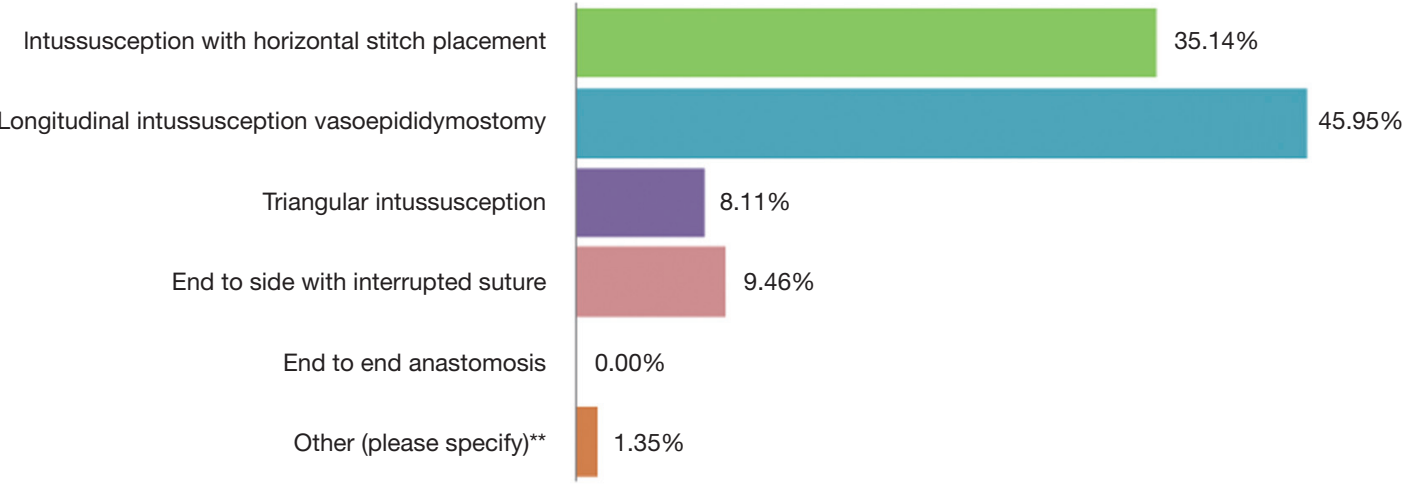

*Other was selected by 2 respondents for epididymotomy technique who reported using a 30-gauge needle or microknife

${ }^{\star \star}$ Other was selected by 1 respondent noting that their technique varies with each operation

Figure 2 Surgical techniques are employed to create epididymotomy and anastomosis. (A) Preferred epididymotomy technique; (B) preferred anastomotic technique.

\section{Discussion}

VR remains a common surgical procedure among men seeking fertility. Despite the rise of ART, VR remains a feasible, effective and typically permanent option for the male partner (10). The operative experience required to perform successful VR, particularly VE, is considerable. Consequently, undertaking an investigation of rates of VR and VE specifically is critical due to the fact that increased microsurgical technique experience is predictive of $\mathrm{VE}$ operative success (9). Despite its technically challenging nature, Grober et al. identified that VE has emerged as an effective VR option for men with obstructive intervals (OI) greater than 10 years (11). Namekawa et al. confirmed these findings and identified that VE outcomes have steadily improved over the past decade in appropriately selected patients (3). Although the number of urologists offering VR is increasing, limited work has been undertaken to characterize its learning curve and the management practices of this expanding provider pool.

We identified notable differences in VE operative volume when stratifying survey participants based on the number of overall VR performed. Our data highlighted that surgeons who undertake higher VR volume were more likely to perform VE. Specialists may be receiving more challenging patients if other providers have identified a patient with a potential need for VE. Practitioners with lower volume or less VE experience may wish to refer complex VR cases to providers with more microsurgical experience, since factors such as ruling out epididymal obstruction are difficult to determine pre-operatively $(3,12,13)$. Providers with increased experience in both VE and VV produce 
A

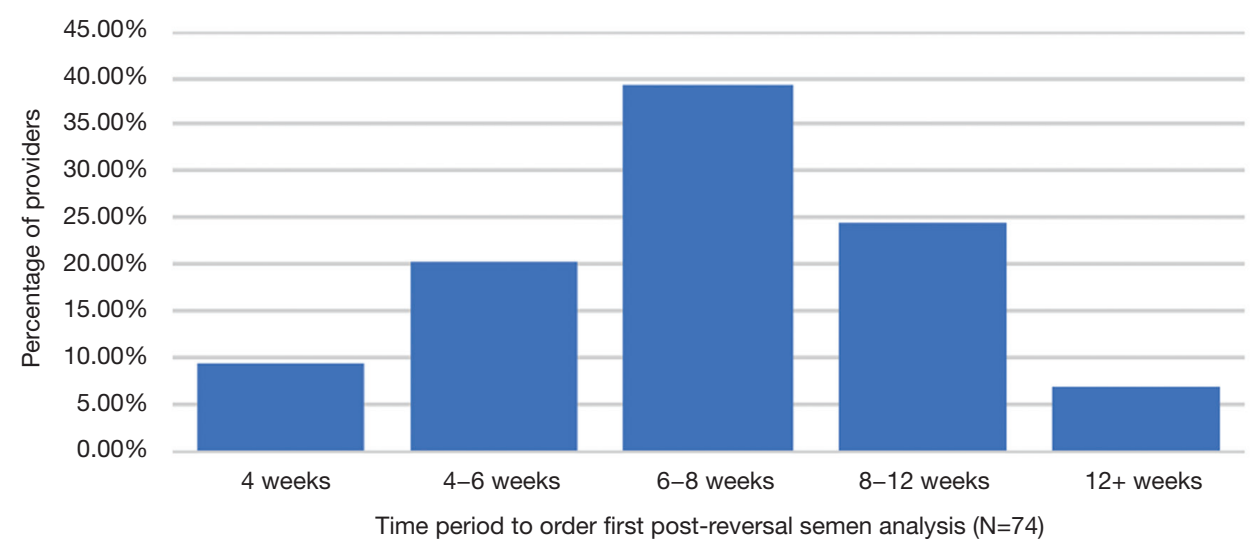

B

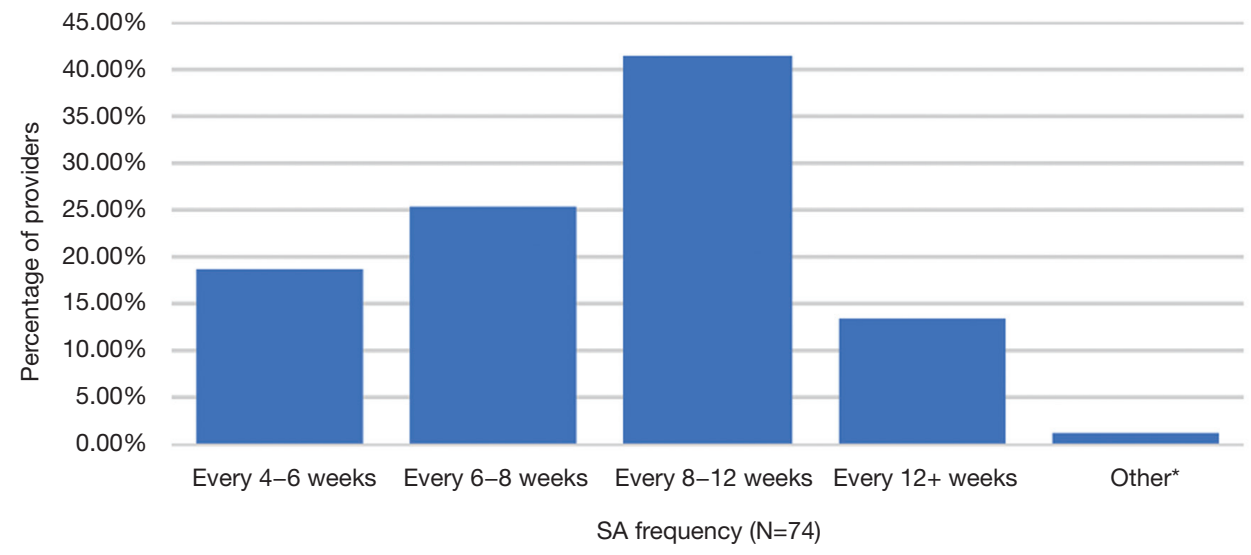

*One respondent indicated SA monitoring at 1, 3, 6, and 12 months post-VR

Figure 3 Overview of preferred time period to order first post-reversal semen analysis (SA) (A) and SA frequency in the setting of subpar post reversal SA parameters (B). VR, vasectomy reversal.

significantly better patency rates, particularly in patients with long OI $(14,15)$. A 2015 review surveying high-volume microsurgeons identified an odds ratio of 3.8 , in reference to post-operative patency, in favor of patients operated on by practitioners with a higher volume (16). Providers with more VE experience have the requisite skill level to perform VE and ensure comparable outcomes between cases needing VV and VE (11). Lastly, providers with increased VR experience overall might have the necessary acumen to intraoperatively assess when $\mathrm{VE}$ is required based on sperm findings. Currently, there are guidelines to suggest when $\mathrm{VE}$ or $\mathrm{VV}$ are required but practices are not standardized $(8,17)$. Providers with higher VR volume and operative experience may be therefore be better positioned to appropriately identify which VR technique will increase the likelihood of success.

Our results demonstrate heterogeneity in the adjunctive therapies and SA protocols that providers employ to manage cases with subpar post-VR SA results. Most providers pursue some form of medical management in this setting. The majority of respondents manage postoperative inflammation through NSAIDs or steroids. Currently, there are no guidelines to dictate the optimal VR postoperative course and the heterogeneity of responses 
Table 1 Ranked preferences of adjunctive therapies employed by survey respondents in the setting of a subpar post-reversal semen analysis

\begin{tabular}{lccccc}
\hline Adjunctive therapies & $1, \mathrm{n}(\%)$ & $2, \mathrm{n}(\%)$ & $3, \mathrm{n}(\%)$ & $4, \mathrm{n}(\%)$ & $5, \mathrm{n}(\%)$ \\
\hline NSAIDs & $39(52.7)$ & $15(20.3)$ & $9(12.2)$ & $10(13.5)$ & $1(1.4)$ \\
Steroids & $17(23.0)$ & $28(37.8)$ & $9(12.2)$ & $6(8.1)$ & $14(18.9)$ \\
Repeat surgery & $2(2.7)$ & $5(6.8)$ & $23(31.1)$ & $30(40.5)$ & $14(18.9)$ \\
Refer to IVF & $7(9.5)$ & $19(25.7)$ & $29(39.2)$ & $17(23.0)$ & $2(2.7)$ \\
None & $9(12.2)$ & $7(9.5)$ & $4(5.4)$ & $11(14.9)$ & $43(58.1)$ \\
\hline
\end{tabular}

NSAIDs, nonsteroidal anti-inflammatory drugs; IVF, in vitro fertilization.

pertaining to specific pain management reflects providerdependent variation. Survey responses indicate that referral to IVF is preferred over attempts to repeat surgery after a suboptimal VR. Intraoperative sperm cryopreservation is often recommended in the setting of prolonged OI or other factors that risk VR failure (18). However, this is an area of active investigation with some data indicating that $\mathrm{VE}$ may be more appropriate than IVF (19). Nevertheless, in our study, repeat surgery is less commonly pursued, given the challenges posed by undertaking redo VR (20). Sixtyeight percent of participants in this study highlighted that they preferred to monitor SA in the setting of subpar postreversal SA parameters at an interval of 6-12 weeks. This SA timeline aligns with prior recommendations put forth by Belker et al. who identified an optimal interval of 2 to 3 months (21).

Another core component of this investigation involved understanding surgical techniques employed by providers who undertake VR. Eighty-nine percent of our respondents indicated that longitudinal intussusception VE (LIVE) and triangular intussusception VE (TIVE) techniques were the preferred anastomotic approach. These results are consistent with prior work investigating VE surgical techniques. Schiff et al. highlighted that LIVE and TIVE techniques yield significantly lower failure rates compared to other techniques such as end-to-end (EE) and end-toside (ES) anastomosis (22). Late shutdown, or azoospermia after an initially successful $\mathrm{VE}$, is one of the major causes of failure, but LIVE utilizes fewer sutures and thus constitutes a simpler, safer, and more successful technique than those previously used (9). Our results highlight that providers offering $\mathrm{VE}$ are undertaking the procedure in line with best practices that have been previously established in larger prospective studies investigating VE outcomes.

There are limitations inherent in the design of this study. Our survey was confined to members of the SMRU, a group of largely fellowship-trained infertility specialists, and therefore this study doesn't necessarily represent the population of general urologists who perform VR. Moreover, our small sample of 74 (23.1\%) respondents may comprise the more active or specialized surgeons within SMRU. Due to this selection bias, the generalizability of our findings is limited and may not capture the heterogeneity of surgical techniques and practices surrounding VR and VE as well as the practice patterns of SMRU members who did not respond to this survey. Although our response rate was $23.1 \%$, this either aligns with or improves upon rates observed in prior investigations with this form of study design $(23,24)$. Recall bias may also interfere with our stratification of practitioners based on case volume and VE proportion. This could impact our characterization of practices based on experience. Our measurement of experience was case volume per year rather than lifetime case volume, which may be a better indicator of experience despite suffering from greater recall bias. Nevertheless, our study provides valuable insight into intraoperative and postoperative practice patterns and is part of the incremental progress towards a standardized management plan for VR.

\section{Conclusions}

The success of VE in epididymal obstruction, its relative cost-effectiveness, and its utility in repeat VR distinguish it as a powerful technique for complicated VR patients. Our study aligns with prior work that has identified surgical experience as an integral factor to the success of $\mathrm{VE}$, and that this procedure should be reserved for providers with extensive microsurgical experience. We identified several variations in provider VR experience, operative technique 
and postoperative management. The heterogeneity of respondents' intraoperative and postoperative practices demonstrates the need for future efforts to standardize VR related protocols. Further understanding of the precise factors that indicate $\mathrm{VE}$ over $\mathrm{VV}$ and identification of best practices in postoperative management are required in order to help facilitate the development of these guidelines.

\section{Acknowledgments}

Funding: This work is supported in part by NIH grant K12 DK0083014, the Multidisciplinary K12 Urologic Research (KURe) Career Development Program (N Thirumavalavan is a K12 Scholar).

\section{Footnote}

Conflicts of Interest: Dr. Lipshultz: American Medical Systems (speaker), AbbVie (consultant), Lipocine (consultant), Aytu Bioscience (consultant), Endo Pharmaceuticals (speaker/ consultant). The other authors have no conflicts of interest to declare.

Ethical Statement: The authors are accountable for all aspects of the work in ensuring that questions related to the accuracy or integrity of any part of the work are appropriately investigated and resolved.

\section{References}

1 Patel AP, Smith RP. Vasectomy reversal: a clinical update. Asian J Androl 2016;18:365-71.

2. Sandlow JI, Nagler HM. Vasectomy and vasectomy reversal: important issues. Preface. Urol Clin North Am 2009;36:xiii-xiv.

3. Namekawa T, Imamoto T, Kato M, et al. Vasovasostomy and vasoepididymostomy: Review of the procedures, outcomes, and predictors of patency and pregnancy over the last decade. Reprod Med Biol 2018;17:343-55.

4. Dickey RM, Pastuszak AW, Hakky TS, et al. The evolution of vasectomy reversal. Curr Urol Rep 2015;16:40.

5. Scovell JM, Mata DA, Ramasamy R, et al. Association between the presence of sperm in the vasal fluid during vasectomy reversal and postoperative patency: a systematic review and meta-analysis. Urology 2015;85:809-13.

6. Smith RP, Khanna A, Kovac JR, et al. The significance of sperm heads and tails within the vasal fluid during vasectomy reversal. Indian J Urol 2014;30:164-8.

7. Belker AM, Thomas AJ, Fuchs EF, et al. Results of 1,469 microsurgical vasectomy reversals by the Vasovasostomy Study Group. J Urol 1991;145:505-11.

8. Silber SJ. Microscopic vasectomy reversal. Fertil Steril 1977;28:1191-202.

9. Chan PT. The evolution and refinement of vasoepididymostomy techniques. Asian J Androl 2013;15:49-55.

10. Shridharani A, Sandlow JI. Vasectomy reversal versus IVF with sperm retrieval: which is better? Curr Opin Urol 2010;20:503-9.

11. Grober ED, Karpman E, Fanipour M. Vasectomy reversal outcomes among patients with vasal obstructive intervals greater than 10 years. Urology 2014;83:320-3.

12. Kolettis PN. Is physical examination useful in predicting epididymal obstruction? Urology 2001;57:1138-40.

13. Chawla A, O'Brien J, Lisi M, et al. Should all urologists performing vasectomy reversals be able to perform vasoepididymostomies if required? J Urol 2004;172:1048-50.

14. Boorjian S, Lipkin M, Goldstein M. The impact of obstructive interval and sperm granuloma on outcome of vasectomy reversal. J Urol 2004;171:304-6.

15. Niederberger C. Re: Vasectomy reversal outcomes among patients with vasal obstructive intervals greater than 10 years. J Urol 2014;192:504.

16. Ramasamy R, Mata DA, Jain L, et al. Microscopic visualization of intravasal spermatozoa is positively associated with patency after bilateral microsurgical vasovasostomy. Andrology 2015;3:532-5.

17. Kolettis PN, Burns JR, Nangia AK, et al. Outcomes for vasovasostomy performed when only sperm parts are present in the vasal fluid. J Androl 2006;27:565-7.

18. Matthews GJ, McGee KE, Goldstein M. Microsurgical reconstruction following failed vasectomy reversal. J Urol 1997;157:844-6.

19. Schrepferman CG, Carson MR, Sparks AET, et al. Need for sperm retrieval and cryopreservation at vasectomy reversal. J Urol 2001;166:1787-9.

20. Hernandez J, Sabanegh ES. Repeat vasectomy reversal after initial failure: overall results and predictors for success. J Urol 1999;161:1153-6.

21. Belker A. Vasectomy Reversal. In: Schill WB, Comhaire F, Hargreave TB. editors. Andrology for the Clinician. Berlin, Heidelberg: Springer, 2006:119-21.

22. Schiff J, Chan P, Li PS, et al. Outcome and late 
failures compared in 4 techniques of microsurgical vasoepididymostomy in 153 consecutive men. J Urol 2005;174:651-5; quiz 801.

23. Butaney M, Thirumavalavan N, Hockenberry MS, et al. Variability in penile duplex ultrasound international practice patterns, technique, and interpretation: an

Cite this article as: Pathak US, Balasubramanian A, Beilan JA, Butaney M, Tatem AJ, Thirumavalavan N, Lipshultz LI. Vasoepididymostomy: an insight into current practice patterns. Transl Androl Urol 2019;8(6):728-735. doi: 10.21037/ tau.2019.11.04 anonymous survey of ISSM members. Int J Impot Res 2018;30:237-42.

24. Kim HL, Hollowell CM, Patel RV, et al. Use of new technology in endourology and laparoscopy by american urologists: internet and postal survey. Urology 2000;56:760-5. 
Supplementary file 1 Overview of anonymous questionnaire sent to Society for Male Reproduction and Urology (SMRU) members

1. Please indicate if you would like to participate?
A. Yes
B. No

2. How many vasectomy reversals do you perform per year?
A. $0-10$
B. $11-20$
C. $21-30$
D. $31-40$
E. $41-50$
F. $51-60$
G. $60+$

3. What percent of these do you estimate involve a VE on at least one side?
A. $0-20 \%$
B. $21-40 \%$
C. $41-60 \%$
D. $61-80 \%$
E. $81-100 \%$

4. What technique do you use to create your epididymotomy?
A. Two 10-0 sutures into the tubule, with an incision to create the epididymotomy
B. No sutures; a blade/microknife used to enter the tubule
C. Microscissors to open an ellipse in the tubule
D. Using a $10-0$ or 11-0 suture to pull through and create a hole in the tubule
E. Other (please specify)

5. What is your most commonly performed anastomotic technique?
A. Intussusception with horizontal stitch placement
B. Intussusception with vertical stitch placement (longitudinal intussusception vasoepididymostomy)
C. Triangular intussusception
D. End to side with interrupted suture
E. End to end anastomosis
F. Other (please specify)

6. At what time period do you order your first post-reversal semen analysis?
A. 4 weeks
B. 4-6 weeks
C. 6-8 weeks
D. $8-12$ weeks
E. $12+$ weeks
F. Other (please specify)

7. What adjunctive therapies do you employ in the setting of a subpar post-reversal semen analysis? (rank 1-5 from your first maneuver to your last maneuver)
A. NSAIDS [1-5]
B. Steroids [1-5]
C. Repeat Surgery [1-5]
D. Refer to IVF [1-5]
E. None [1-5]

8. How often do you follow SA's in the setting of subpar post-reversal SA parameters?
A. Every 4-6 weeks
B. Every 6-8 weeks
C. Every 8-12 weeks
D. Every $12+$ weeks
E. Other (please specify) 